\title{
Comparison between C1-2 Fixation with and without Supplemental Posterior Wiring
}

\author{
Mai $\operatorname{Tran}^{1}$ Rishi Wadhwa ${ }^{2}$ John Ziewacz ${ }^{2}$ Praveen Mummaneni ${ }^{2}$ Dean Chou $^{2}$ \\ ${ }^{1}$ Miller School of Medicine, University of Miami, Miami, Florida, \\ United States \\ ${ }^{2}$ Department of Neurological Surgery, University of California San \\ Francisco, San Francisco, California, United States \\ Address for correspondence Mai Tran, BS, 12520 SW 94th Lane, \\ Miami, FL 33186, United States (e-mail: mttran@med.miami.edu). \\ Evid Based Spine Care J 2014;5:12-15.
}

\begin{abstract}
Keywords

- SRC

- posterior wiring

- Harms technique

- atlantoaxial instability

- C1

- C2

- fusion

- screw rod construct
\end{abstract}

Study Design Retrospective analysis.

Clinical Question Is there a difference between the screw-rod construct (SRC) procedure without wiring and the SRC procedure with wiring with respect to fusion, implant failure, reoperation, donor-site morbidity, and complication rates?

Patients and Methods We performed a retrospective analysis of 26 patients who underwent C1-2 fixation between 2004 and 2012 (SRC with wiring and structural bone graft, 13 patients; SRC with autograft but without wiring, 13 patients). Fusion was assessed using dynamic $X$-rays in all patients and computed tomographic scans in selected cases. Pseudoarthrosis was confirmed during reoperation.

Results The mean follow-up time was 2 years and 5 months for the SRC without wiring group and 2 years and 1 month for the SRC with wiring group. Patients with less than 1 year follow-up time were excluded. The fusion rate, implant failure rate, and reoperation rates for the SRC without wiring group were 92,8 , and $8 \%$, respectively. The fusion, implant failure, and reoperation rates for the SRC with wiring group were 100,0 , and $0 \%$, respectively. There were no donor-site morbidities or complications in either group (both $0 \%$ ). There were no differences in parameters we examined between the two groups ( $p>0.05$ for each rate, Fisher exact test).

Conclusions The results suggest that supplementing the SRC procedure with wiring may increase fusion rate, but this difference is not statistically significant. Although the sample size was small, there was not a significant discrepancy in outcomes between the two groups at an average follow-up of 2 years.

\section{Study Rationale and Context}

When treating atlantoaxial stability, the posterior C1 lateral mass screw and $\mathrm{C} 2$ pars or pedicle screw construct (screw-rod construct [SRC]) with onlay autograft have yielded high-fusion rates. ${ }^{1}$ Some surgeons supplement the SRC procedure with posterior wiring using structural

received

July 8,2013

accepted after revision

February 10, 2014

bone graft (Brooks, Gallie, Sonntag), which increases operative time and expense.

\section{Clinical Question}

Is there a difference between the SRC procedure without wiring and the SRC procedure with supplemental wiring with

(c) 2014 Georg Thieme Verlag KG Stuttgart · New York
DOI http://dx.doi.org/ 10.1055/s-0034-1371972. ISSN 1663-7976. 


\begin{tabular}{|l|l|}
\hline Final class of evidence (CoE)-treatment & Yes \\
\hline Study Design & \\
\hline RCT & \\
\hline Cohort & $\mathrm{X}$ \\
\hline Case-control & \\
\hline Case series & \\
\hline Methods & \\
\hline Concealed allocation (RCT) & \\
\hline Intention to treat (RCT) & \\
\hline $\begin{array}{l}\text { Blinded/independent evaluation } \\
\text { of primary outcome }\end{array}$ & \\
\hline F/U $\geq 85 \%$ & \\
\hline Adequate sample size & \\
\hline Control for confounding & \\
\hline Overall class of evidence & \\
\hline
\end{tabular}

respect to fusion, implant failure, reoperation, donor-site morbidity, and complication rates?

\section{Patients and Methods}

\section{Study Design}

Retrospective analysis.

\section{Inclusion Criteria}

Patients who underwent the SRC procedure without or with wiring for C1-2 fixation and had a minimum of 1-year follow-up.

\section{Exclusion Criteria}

Patients who underwent other procedures than the SRC procedure without or with wiring for C1-2 fixation or did not have a minimum of 1-year follow-up (-Fig. 1).

\section{Patient Population and Interventions Compared}

- Thirteen patients underwent the SRC procedure without wiring (five females, eight males) (-Fig. 1, - Table 1).

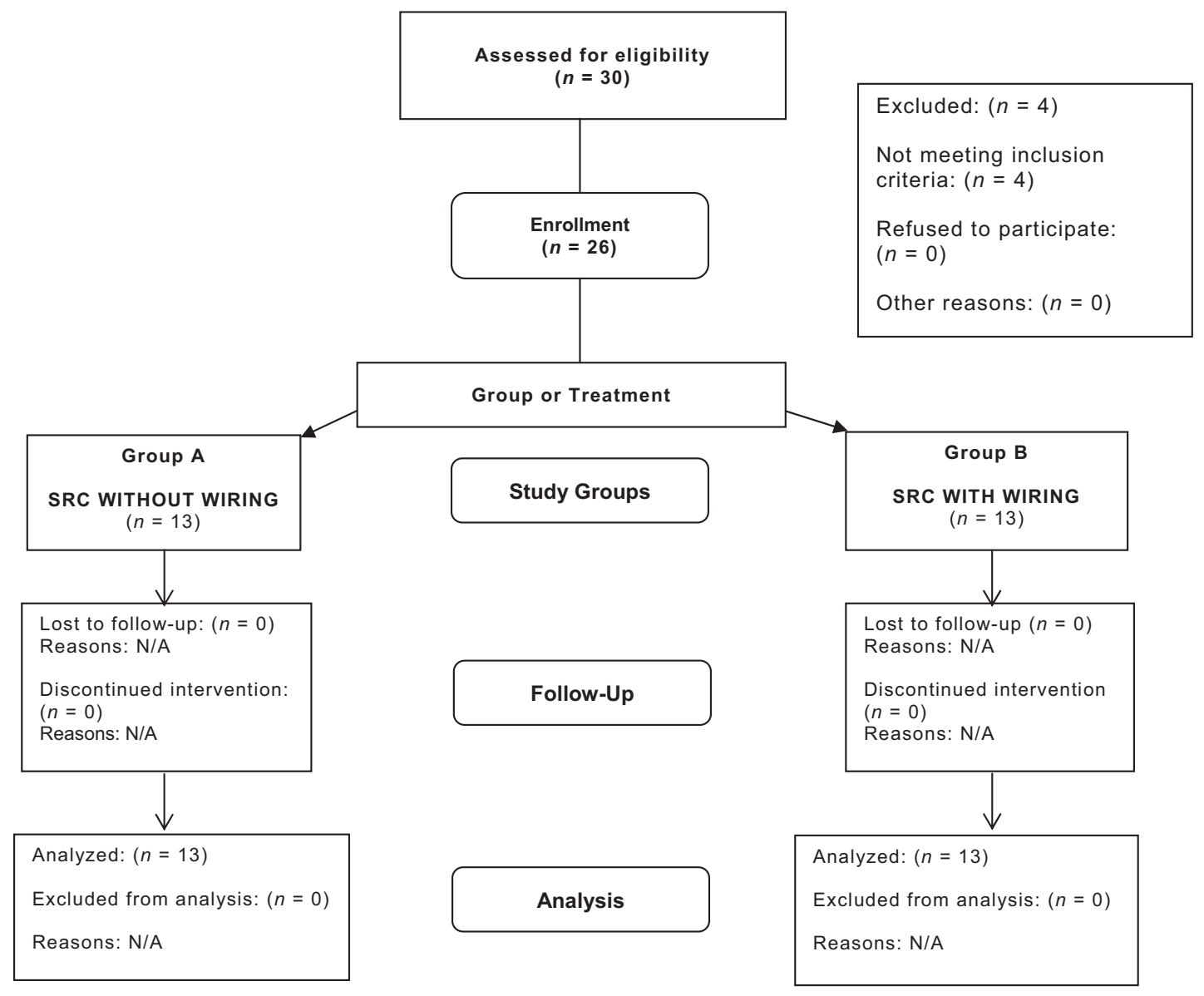

Fig. 1 After eligibility assessment, 26 patients were enrolled in the SRC without wiring vs. SRC with wiring study: 13 in each group; 0 lost to follow-up. SRC, screw-rod construct. 
14 C1-2 Fixation with and without Supplemental Posterior Wiring Tran et al.

Table 1 Demographic and baseline characteristics of intervention groups

\begin{tabular}{|l|l|l|}
\hline & $\begin{array}{l}\text { Treatment A: } \\
\text { SRC without wiring, } \boldsymbol{n}=13\end{array}$ & $\begin{array}{l}\text { Treatment B: } \\
\text { SRC with wiring, } \boldsymbol{n}=\mathbf{1 3}\end{array}$ \\
\hline Age $(\mathrm{y}$, mean \pm SD) & $(65 \pm 22.2)$ & $(65 \pm 18.7)$ \\
\hline Female $(n, \%)$ & $5(38)$ & $6(46)$ \\
\hline Male $(n, \%)$ & $8(62)$ & $7(54)$ \\
\hline Osteoporosis $(n, \%)$ & $2(15)$ & $1(8)$ \\
\hline Acute $(n, \%)$ & $1(8)$ & $2(15)$ \\
\hline Chronic $(n, \%)$ & $12(92)$ & $11(85)$ \\
\hline Pathology & & $3(23)$ \\
\hline RA pannus $(n, \%)$ & $4(31)$ & $9(69)$ \\
\hline Fracture $(n, \%)$ & $6(46)$ & $1(8)$ \\
\hline C1-2 transverse ligamentous injury $(n, \%)$ & $3(23)$ & $0(0)$ \\
\hline Loss to follow-up $(n, \%)$ & $0(0)$ & \\
\hline
\end{tabular}

Abbreviations: RA, rheumatoid arthritis; SD, standard deviation; SRC, screw-rod construct.

Note: $p$ values for between group differences as determined by the Fisher exact test for categorical measures.

- Thirteen patients underwent the SRC procedure with wiring (six females, seven males) (-Fig. 1, - Table 1).

- In the SRC procedure, a screw is inserted bilaterally into the C1 lateral masses and the $\mathrm{C} 2$ pars interarticularis or translaminar $^{2}$; the $\mathrm{C} 1$ and $\mathrm{C} 2$ screws are then connected via a rod. ${ }^{3}$

- In Sonntag wiring technique, a wire is passed under the posterior $\mathrm{C} 1$ arch from inferior to superior ${ }^{4}$; a notched graft is then placed over the $\mathrm{C} 2$ spinous process and wedged underneath the posterior $\mathrm{C} 1 \mathrm{arch}^{4}$; the wire is looped over the graft, placed around the C2 spinous process, and seated in notches that were created on the inferior aspect of both sides of the spinous process. ${ }^{4}$

- Grafts used were iliac crest grafts (21 patients), fibular allograft ( 1 patient), laminectomy bone ( 3 patients), and combined C2 spinous process and C1 laminectomy bones (1 patient).

- To control for confounders, osteoporosis rate, acute/chronic condition rate, and pathology (RA pannus, fracture, and C12 transverse ligamentous injury) rates were investigated.

\section{Outcomes}

- Fusion: Absence of motion on flex-ex films and the presence of bridging bone on computed tomographic or plain films.

- Implant failure: Implant pull-out, loosening, or separation.

- Reoperation: Having to undergo another operation because of pseudoarthrosis or implant failure.

- Donor-site morbidity: Complications at the site in which the bone graft was removed.

- Complication: Problems that arise as a result of the surgery such as vertebral artery, neurological injury, infection, etc.

\section{Analysis}

- Fisher exact test.

Additional methodological and technical details are provided in the online supplementary material.

\section{Results}

- Total patients analyzed, average age (age range) in years, mean follow-up time: SRC without wiring group $=13$ patients, 65 (17-91), 2 years and 5 months, respectively; SRC with wiring group $=13$ patients, 65 (30-92), 2 years and 1 month, respectively.

- Osteoporosis rate: SRC without wiring group $=15 \%$; SRC with wiring group $=8 \%$.

- Acute/chronic condition rate: SRC without wiring group $=8 / 92 \%$; SRC with wiring group $=15 / 85 \%$.

- Pathology (RA pannus, fracture, and C1-2 transverse ligamentous injury) rates: SRC without wiring group $=31$, $46,23 \%$, respectively; SRC with wiring group $=23,69$, and $8 \%$, respectively.

- Fusion, implant failure, reoperation rate: SRC without wiring group $=92,8$, and $8 \%$ respectively; SRC with wiring group $=100,0$, and $0 \%$, respectively.

- No donor-site morbidities or complications in either group (0\%).

- No differences in any of the parameters examined between the two groups even when controlling for confounders $(p>0.05$, Fisher exact test) ( - Table 2 ).

\section{Discussion}

\section{Strengths}

- One of the only comparisons between SRC without wiring and SRC with wiring.

- Minimum of 1-year follow-up time.

- Patients were operated at a major academic center.

- Even numbers of patients in both the groups.

- Statistical significance $=p<0.05$ : minimizes the likelihood that the results were due to chance. 
Table 2 Results

\begin{tabular}{|l|l|l|l|}
\hline & SRC without wiring group & SRC with wiring group & Fisher exact test \\
\hline Number of patients analyzed & 13 & 13 & N/A \\
\hline Average follow-up time & 2 y 5 mo & 2 y 1 mo & \\
\hline Fusion rate, \% & 92 & 100 & $p>0.05$ \\
\hline Implant failure rate, \% & 8 & 0 & $p>0.05$ \\
\hline Reoperation rate, \% & 8 & 0 & $p>0.05$ \\
\hline Donor-site morbidity rate, \% & 0 & 0 & $p>0.05$ \\
\hline Complication rate, \% & 0 & 0 & $p>0.05$ \\
\hline
\end{tabular}

Abbreviations: N/A, not applicable; SRC, screw-rod construct.

Note: No one was lost to follow-up.

- Control for the following potential confounders: osteoporosis, acute/chronic condition, and pathology (RA pannus, fracture, and C1-2 transverse ligamentous injury).

\section{Limitations}

- Retrospectively collected data

- Small sample size

- Not randomized

- Not blinded

\section{Clinical Relevance and Impact}

- Supplemental wiring may not provide significant benefit to the patients with respect to the parameters examined.

- The screws and rods themselves may provide sufficient support.

- Because performing the posterior wiring technique increases operative time and expense, the addition of posterior wiring should ultimately be at the surgeon's discretion, taking into account bone quality, fusion potential, and overall confidence in the initial C1-2 construct obtained with only the SRC procedure.

\section{Summary and Conclusions: Key Points}

- Osteoporosis, acute/chronic condition, and pathology (RA pannus, fracture, and C1-2 transverse ligamentous injury) rates were not confounders nor associated with higher reoperation rates.

- There were no significant differences in the fusion, implant failure, reoperation, donor-site morbidity, and complication rates between the SRC without wiring and with wiring groups.

\section{Acknowledgement}

This study was approved by the institutional Committee on Human Research (CHR) at the University of California San Francisco (UCSF).

Conflict of Interest

- Mai Tran, Rishi Wadhwa, and John Ziewacz: None.

- Praveen Mummaneni: Depuy, Globus, 2N LLC.

- Dean Chou: Depuy, Globus, Stryker.

\section{References}

1 Elliott RE, Tanweer O, Boah A, et al. Atlantoaxial Fusion with Screw-Rod Constructs: Meta-Analysis and Review of Literature. World Neurosurg 2012;8750(12):412-417

2 Harms J, Melcher RP. Posterior C1-C2 fusion with polyaxial screw and rod fixation. Spine (Phila Pa 1976) 2001;26(22):2467-2471

3 Sonntag VK. Atlantoaxial stabilization: a minimally invasive alternative. World Neurosurg 2013;80(3-4):315-316

4 Mummaneni PV, Haid RW. Atlantoaxial fixation: overview of all techniques. Neurol India 2005;53(4):408-415

\section{Editorial Perspective}

The publication of this article was controversial among the reviewers. The main concerns were obviously centered around the relatively low sample-size, the recruitment methodology used (were all consecutive C1-2 fusion patients included?) and possible heterogeneity of the compared populations.

That said, after reiterative deliberations, EBSJ has decided in favor of publication as the authors pose an important question: In the age of potential unprecedented quality segmental fixation of a deficient atlantoaxial complex, is the addition of supplemental wiring and posterior onlay or interposition graft still needed, or can other techniques, such as a facet arthrodesis, provide adequate substitution?
While it is obviously desirable to have larger cohorts, especially for comparison studies, more specific or rare conditions with difficult patient recruitments may prompt us to make exceptions as was done here, in order to raise awareness in our surgeon community of other treatment options. That said, this article shows us at-best equivalency of segmental rod constructs alone with the previous posterior arch grafting, but it is clearly premature to use the presented data as a foundation to declare a new standard of care. We hope that the presented study will prompt investigations into more formal determinants of atlantoaxial fusion and efforts at larger scale patient safety and outcomes reporting of these patients. 